\title{
RELAY FEEDBACK AUTOTUNING - A POLYNOMIAL DESIGN APPROACH
}

\author{
Roman Prokop, Jiří Korbel and Zdenka Prokopová \\ Faculty of applied informatics \\ Tomas Bata University in Zlín \\ Nad Stráněmi 4511, 76005 Zlín, Czech Republic \\ E-mail: prokop@fai.utb.cz
}

\section{KEYWORDS}

Auto-tuning, Diophantine equation, RPS synthesis, PID controller, relay feedback.

\begin{abstract}
A method of autotuning using an asymmetric relay with hysteresis feedback test is proposed and developed. Then, three parameters for aperiodic first or second order transfer functions can be obtained. After the identification relay experiment, controller parameters are computed through linear diophantine equation in the ring of proper and stable rational functions. This algebraic approach for a traditional 1-DOF feedback structure generates a class of PI or PID controllers. The pole placement principle in the methodology brings a scalar positive "tuning knob" for additional controller tuning. A Matlab-Simulink program implementation was developed for simulation and verification of the studied approach. Two illustrative examples support simplicity and efficiency of the proposed methodology.
\end{abstract}

\section{INTRODUCTION}

Despite of expressive evolution of control hardware, PID controllers remain the main tool in industrial feedback loops and they survived many changes in technology. The practical advantages of PID controllers can be seen in a simple structure, in an understandable principle and in control capabilities. It is widely known that PID controllers are quite resistant to changes in the controlled process without meaningful deterioration of the loop behavior.

However, real industrial processes suffer from many unpleasant drawbacks as non-linearity, complexity and time variance. These features induce difficulties to control their loops properly. Adequate and sufficient tuning of controllers needs to know relevant process parameters. One way how to overcome the mentioned problems consists in automatic tuning of controllers. The development of various autotuning principles was started by a simple symmetrical relay feedback experiment (Aström and Hägglund 1984) for a PID structure. Ultimate gain and ultimate frequency are then used for adjusting of parameters by common known Ziegler-Nichols rules. During the period of more than two decades, many studies have been reported to extend and improve autotuners principles; see e.g. (Aström and Hägglund 1995; Ingimundarson and Hägglund 2000; Majhi and Atherton 1998; Morilla at al. 2000). The extension in an experimental phase was performed in (Yu 1999; Pecharromán and Pagola 2000; Kaya and Atherton 2001) by an asymmetry and hysteresis of a relay, see (Thyagarajan and Yu 2002), and experiments with asymmetrical and dead-zone relay feedback are reported in (Vítečková and Víteček 2004; Vyhlídal 2000). Also, various control design principles and rules can be investigated in mentioned references. Nowadays, almost all commercial industrial PID controllers provide the feature of autotuning. This paper is focused on a novel combination for autotunig method of PI and PID controllers. The method combines an asymmetrical relay identification experiment and a control design which is based on a pole-placement principle. The pole placement problem is formulated through a Diophantine equation and it is tuned by an equalization setting proposed in (Gorez and Klán 2000).

\section{PROCESS PARAMETERS IDENTIFICATION}

System identification of the process parameters is a crucial point for many auto-tuning principles. The identification rules with relay in feedback loops can utilize various types of relays. The relay feedback proposed by Aström in 1984 used a symmetrical relay without hysteresis. The identification procedure with a relay in the feedback loop can utilize various types of relays. The relay feedback proposed by Aström in 1984 utilizes symmetrical relay without hysteresis. This procedure gives the ultimate parameters of the process and control design may follow. Unfortunately, the process gain must be known in advance because the symmetrical relay test cannot identify it. On the other hand, the process gain can be obtained during the relay feedback test when an asymmetrical relay is utilized. A typical data response from the relay experiment can be seen in Figure 1.

In this paper, an asymmetric relay with hysteresis is used. It enables to identify the parameters of the transfer function, such as proportional gain, time constant, as well as time delay term. Time delay is approximated by Pade approximation before the algebraic controller design. 


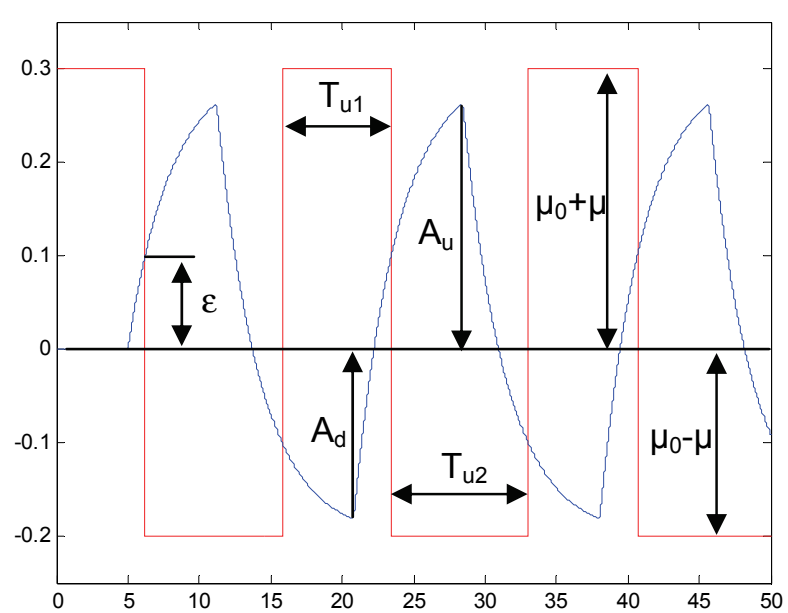

Figure 1: Relay feedback test of stable process

\section{First order system}

The most popular and simplest approximation of aperiodic industrial processes can be characterized by the first order transfer function with time delay (FOPDT) in the form:

$$
G(s)=\frac{K}{T s+1} \cdot e^{-L s}
$$

When the asymmetric relay is used for the relay feedback test, it is shown in Figure 1, the output y converges to the stationary oscillation in one period. These oscillations are characterized by equations (Hang et al. 2001):

$$
\begin{aligned}
& A_{u}=\left(\mu_{0}+\mu\right) \cdot K \cdot\left(1-e^{-\frac{L}{T}}\right)+\varepsilon \cdot e^{-\frac{L}{T}} \\
& A_{d}=\left(\mu_{0}-\mu\right) \cdot K \cdot\left(1-e^{-\frac{L}{T}}\right)-\varepsilon \cdot e^{-\frac{L}{T}} \\
& T_{u 1}=T \cdot \ln \frac{2 \cdot \mu \cdot K \cdot e^{\frac{L}{T}}+\mu_{0} \cdot K-\mu \cdot K+\varepsilon}{\mu \cdot K+\mu_{0} \cdot K-\varepsilon} \\
& T_{u 2}=T \cdot \ln \frac{2 \cdot \mu \cdot K \cdot e^{\frac{L}{T}}-\mu_{0} \cdot K-\mu \cdot K+\varepsilon}{\mu \cdot K-\mu_{0} \cdot K-\varepsilon}
\end{aligned}
$$

The proportional gain can be alternatively computed from the equation (Vyhlídal 2000):

$$
K=\frac{\int_{0}^{T_{y}} y(t) d t}{\int_{0}^{T_{y}} u(t) d t}, \quad T_{y}=T_{u 1}+T_{u 2}
$$

The normalized dead time of the process $(\mathrm{L} / \mathrm{T})$ is obtained from (2) or (3) in the form (Hang et al. 2001):

$$
\Theta=\ln \frac{\left(\mu_{0}+\mu\right) \cdot K-\varepsilon}{\left(\mu_{0}+\mu\right) \cdot K-A_{u}}
$$

or

$$
\Theta=\ln \frac{\left(\mu-\mu_{0}\right) \cdot K-\varepsilon}{\left(\mu-\mu_{0}\right) \cdot K+A_{d}}
$$

Next, the time constant can be computer from (4) or (5) by solving these formulas:

$$
T=T_{u 1} \cdot\left(\ln \frac{2 \cdot \mu \cdot K \cdot e^{\Theta}+\mu_{0} \cdot K-\mu \cdot K+\varepsilon}{\mu \cdot K+\mu_{0} \cdot K-\varepsilon}\right)^{-1}
$$

or

$$
T=T_{u 2} \cdot\left(\ln \frac{2 \cdot \mu \cdot K \cdot e^{\Theta}-\mu_{0} \cdot K-\mu \cdot K+\varepsilon}{\mu \cdot K-\mu_{0} \cdot K-\varepsilon}\right)^{-1}
$$

The dead time is $L=T \cdot \Theta$.

\section{Second order system}

The process is described by the second order transfer function with time delay (SOPDT):

$$
G(s)=\frac{K}{(T s+1)^{2}} \cdot e^{-L s}
$$

The process gain can be computed by the same equation (6) as for the first order system.

The time constant and time delay term can be estimated by relations (Vítečková and Víteček 2004)

$$
\begin{aligned}
T & =\frac{T_{y}}{2 \pi} \cdot \sqrt{\frac{4 \cdot K \cdot u_{0}}{\pi \cdot a_{y}}-1} \\
L & =\frac{T_{y}}{2 \pi} \cdot\left[\pi-2 \arctan \frac{2 \pi T}{T_{y}}-\arctan \frac{\varepsilon}{\sqrt{a_{y}^{2}-\varepsilon^{2}}}\right]
\end{aligned}
$$

where $T_{y}=T_{u 1}+T_{u 2}, \quad u_{0}=\mu, \quad a_{y}=\frac{A_{u}+A_{d}}{2}$.

\section{CONTROLLER DESIGN}

The control design is based on the fractional approach (Vidyasagar 1987; Kučera 1993; Prokop and Corriou 1997; Prokop et al. 2002). Any transfer function G(s) of a (continuous-time) linear system is expressed as a ratio of two elements of $R_{P S}$. The set $R_{P S}$ means the ring of (Hurwitz) stable and proper rational functions. Traditional transfer functions as a ratio of two polynomials can be easily transformed into the fractional form simply by dividing, both the polynomial denominator and numerator by the same stable polynomial of the order of the original denominator. 
Then all transfer functions can be expressed by the ratio:

$$
\begin{aligned}
& G(s)=\frac{b(s)}{a(s)}=\frac{\frac{b(s)}{(s+m)^{n}}}{\frac{a(s)}{(s+m)^{n}}}=\frac{B(s)}{A(s)} \\
& n=\max (\operatorname{deg}(a), \operatorname{deg}(b)), \quad m>0
\end{aligned}
$$

All feedback stabilizing controllers according to Figure 2 are given by a general solution of the Diophantine equation:

$$
A P+B Q=1
$$

which can be expressed with $\mathrm{Z}$ free in $\mathrm{R}_{\mathrm{PS}}$ :

$$
\frac{Q}{P}=\frac{Q_{0}-A Z}{P_{0}+B Z}
$$

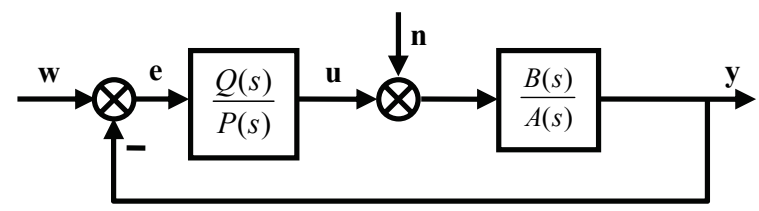

Figure 2: Feedback (1DOF) control loop

Asymptotic tracking is then ensured by the divisibility of the denominator $P$ in (17) by the denominator of the reference $w=\frac{G}{F}$ which is supposed in the form:

$$
F_{w}=\frac{s}{s+m} ; \quad m>0
$$

The set of reference signals with denominator (18) represents all stepwise signals which are most frequent references. The divisibility is achieved by a suitable choice of rational function $\mathrm{Z}$ in (17), see (Prokop et al. 2002).

The control design for first order systems (1) without time delay gives the Diophantine equation (16) in the form:

$$
(T s+1) p_{0}+K q_{0}=s+m
$$

and the general solution is given by:

$$
\begin{aligned}
& P=\frac{1}{T}+\frac{K}{s+m} \cdot Z \\
& Q=\frac{T m-1}{T K}-\frac{T s+1}{s+m} \cdot Z
\end{aligned}
$$

where $\mathrm{Z}$ is free in the ring $\mathrm{R}_{\mathrm{PS}}$. Asymptotic tracking is achieved by the choice:

$$
Z=-\frac{m}{T K}
$$

and the resulting PI controller is in the form:

$$
C(s)=\frac{Q}{P}=\frac{q_{1} s+q_{0}}{s}
$$

where parameters $\mathrm{q}_{1}$ a $\mathrm{q}_{0}$ are given by:

$$
q_{1}=\frac{2 T m-1}{K} \quad q_{0}=\frac{T m^{2}}{K}
$$

Second order systems give the design equation in the form:

$$
(T s+1)^{2} \cdot s \cdot p_{0}+K \cdot\left(q_{2} s^{2}+q_{1} s+q_{0}\right)=(s+m)^{3}
$$

and after similar manipulations the resulting PID controller gives the transfer function:

$$
C(s)=\frac{Q}{P}=\frac{q_{2} s^{2}+q_{1} s+q_{0}}{s\left(s+p_{0}\right)}
$$

with parameters:

$$
\begin{aligned}
& p_{0}=\frac{1}{T^{2}} ; \quad q_{2}=\frac{3 T m-2}{K T} \\
& q_{1}=\frac{3 T^{2} m^{2}-1}{K T^{2}} ; \quad q_{0}=\frac{m^{3}}{K}
\end{aligned}
$$

The scalar parameter $m>0$ seems to be a suitable „tuning knob” influencing control behavior as well as robustness properties of the closed loop system. Some principles and approaches exist for a "good" or "optimal" choice of this parameter. One of them is based on the equalization principle, proposed in Gorez and Klán 2000.

Polynomial control synthesis is based on neglecting of time delay terms. However, ignoring of this term generally can not be acceptable, especially for its higher values. Then a Pade approximation can be used in the form

$$
e^{-L s} \approx \frac{1-\frac{L s}{2}}{1+\frac{L s}{2}}
$$

and naturally, control equation (16) leads to higher orders of control transfer functions. For automatic control design, a program system in Matlab and Simulink with the support of the Polynomial Toolbox 
was developed. Controller formulas then are more complex than (23) and (26) and resulting controllers lose PI or PID structures. Figure 3 illustrates the main menu of the program system.

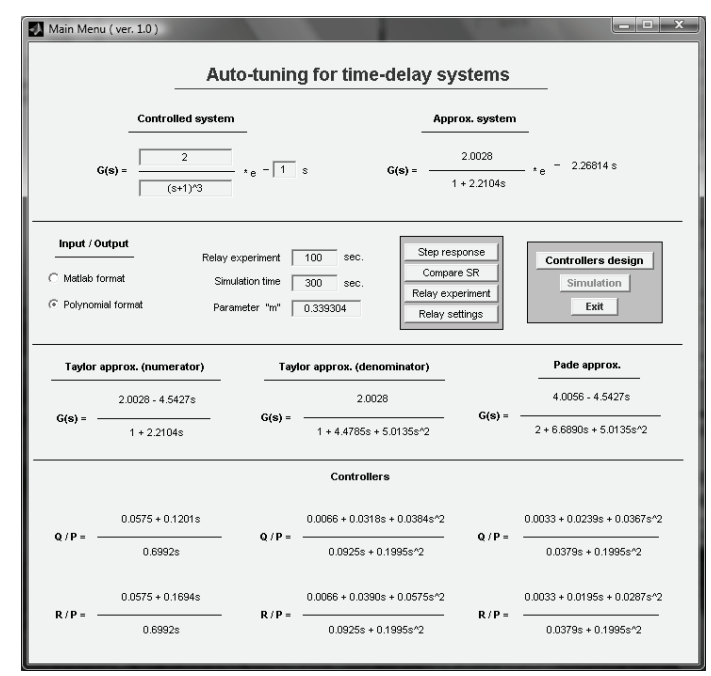

Figure 3: Main menu of the program

\section{SIMULATION EXAMPLES}

Example 1: A third order controlled system with the transfer function:

$$
G(s)=\frac{2}{(2 s+1.1)^{3}} \cdot e^{-2 s}
$$

was identified in the relay feedback loop as a first order model:

$$
\widetilde{G}(s)=\frac{1.5}{4.5 s+1} \cdot e^{-3.8 s}
$$

Step responses of the controlled system and its identified model are compared in Figure 4.

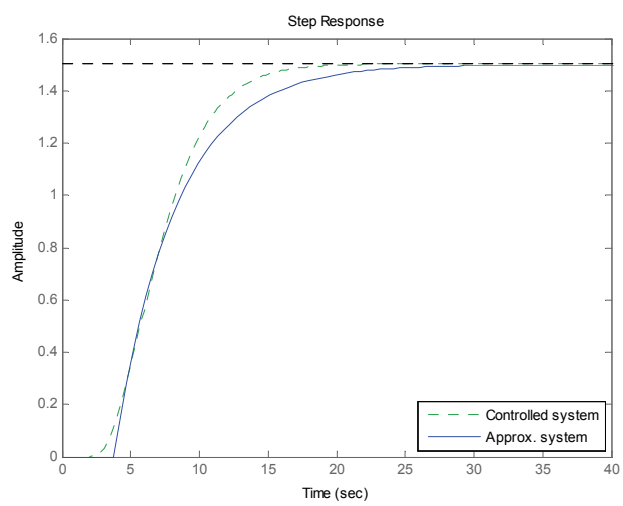

Figure 4: Step responses of systems
The following controller design was performed for two different tuning parameters $m$. Control responses can be seen in Figure 5 and Figure 6.

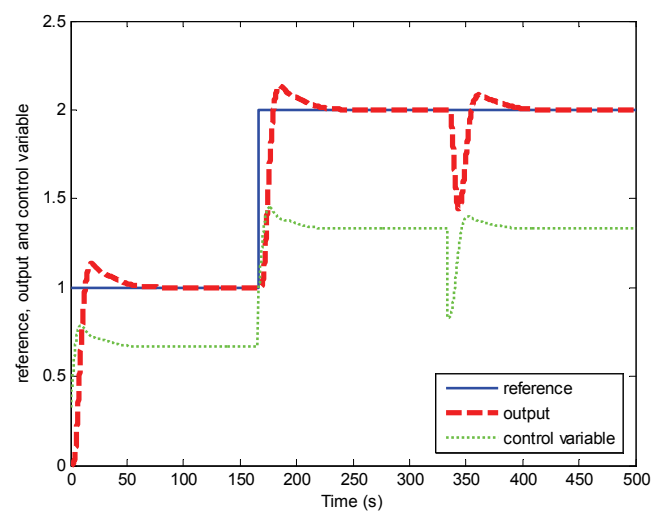

Figure 5: Control response for $\mathrm{m}=0.17$

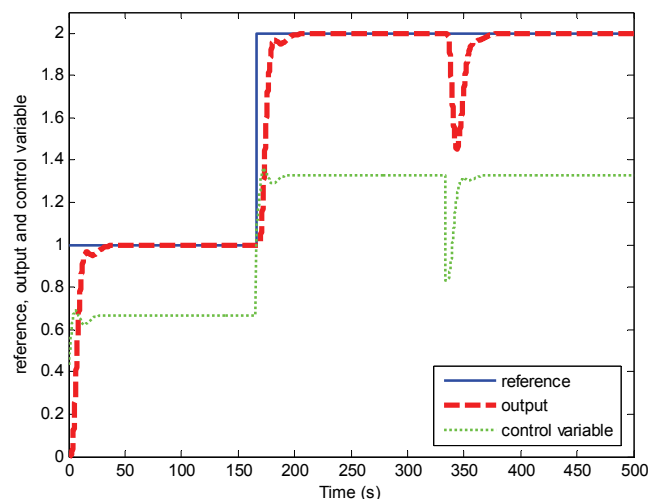

Figure 6: Control response for $\mathrm{m}=0.3$

Example 2: A fourth order controlled system governed by the transfer function:

$$
G(s)=\frac{1.5}{(1.5 s+1)^{4}} \cdot e^{-3 s}
$$

was again approximated in similar way as a first order model:

$$
\widetilde{G}(s)=\frac{1.5}{3.9 s+1} \cdot e^{-5.7 s}
$$

The comparison of step responses is depicted in Figure 7. In (31), two different approaches to the time delay term were considered. The first one represents a simple neglecting of the time delay. The control response is depicted in Figure 8. The second approach utilizing Pade approximation (27) leads to responses shown in Figure 9.

As can be seen in the control responses, the value of the tuning parameter $m>0$ strongly influences the control response, e.g. the increasing values of $m>0$ accelerate the control response but escalate overshoots. 


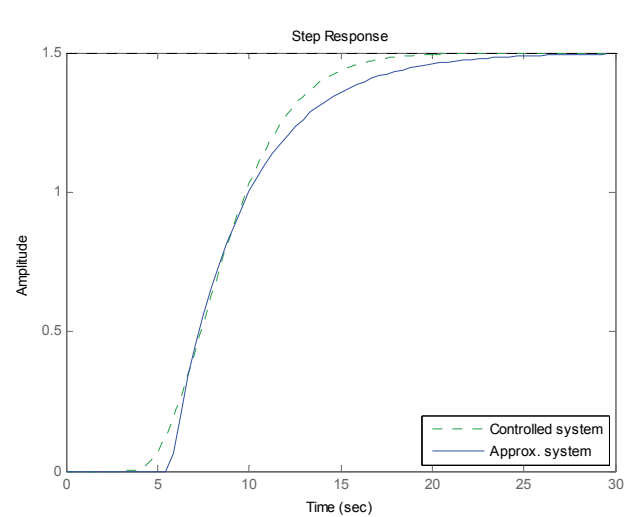

Figure 7: Comparison of step responses (30), (31)

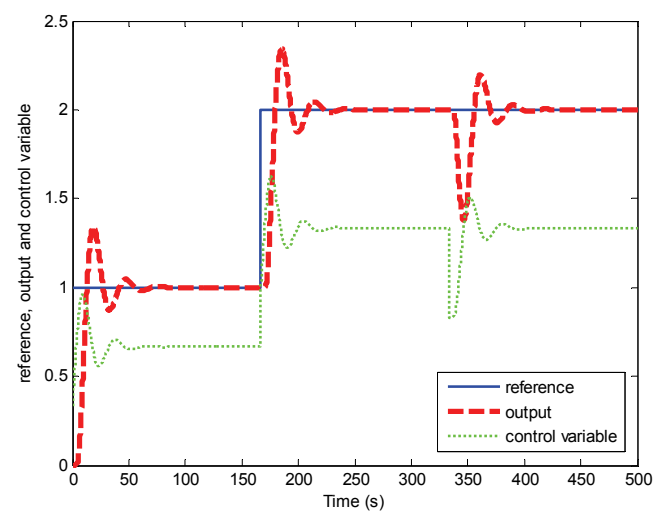

Figure 8: Neglected time delay, $\mathrm{m}=0.19$

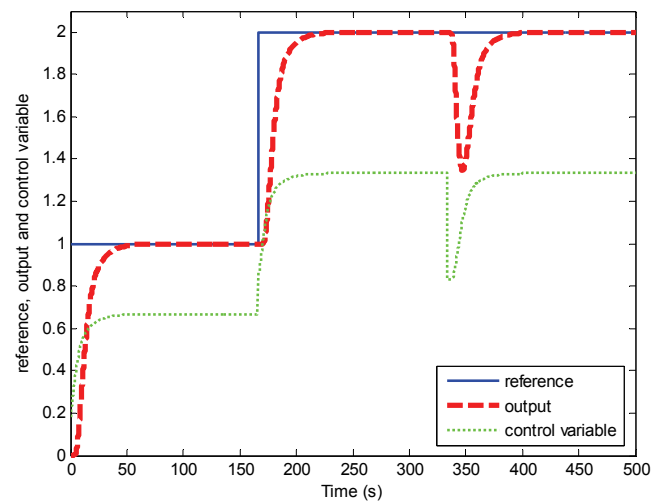

Figure 9: Approximation of time delay, $\mathrm{m}=0.19$

Example 3: A higher order system was simultaneously identified as a first and second order model with transport delay. The controlled system was governed by transfer function:

$$
G(s)=\frac{2}{(2 s+1)^{8}} \cdot e^{-3 s}
$$

After relay feedback experiments, the identification procedure for (1) and (11) gives two transfer functions:

$$
\begin{aligned}
& \widetilde{G}(s)=\frac{2}{7.7 s+1} \cdot e^{-12.9 s} \\
& \widetilde{G}(s)=\frac{2}{(4.4 s+1)^{2}} \cdot e^{-10.7 s}
\end{aligned}
$$

In both cases, time delay terms were approximated by Pade simple formula (27) and then the polynomial control design procedure was performed.

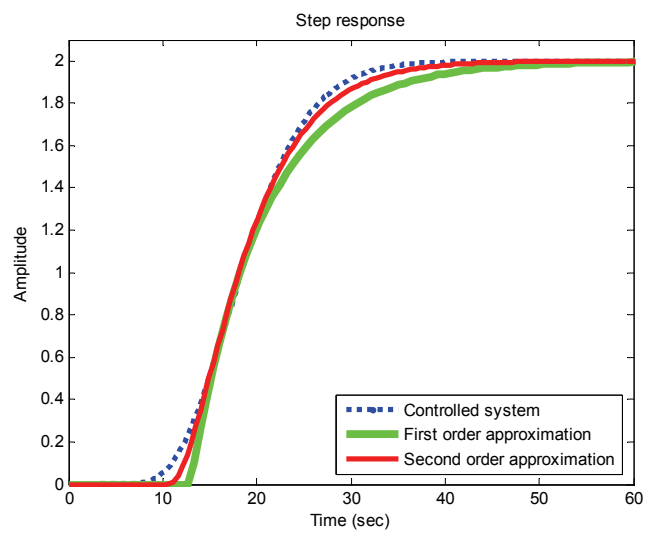

Figure 10: Comparison of step responses (32)-(34)

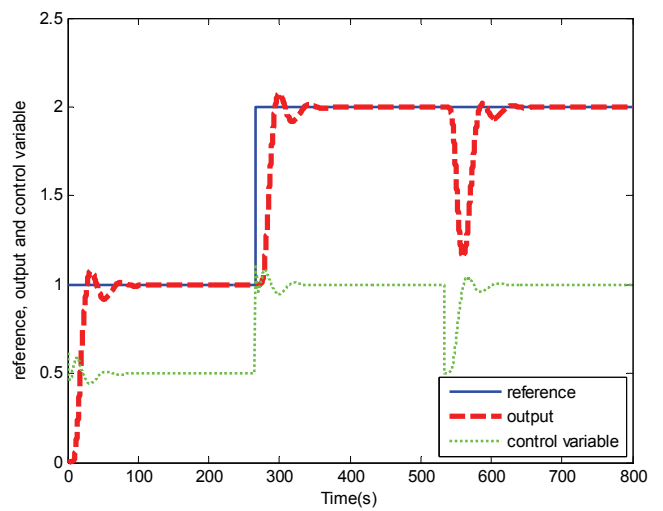

Figure 11: FOPDT model (33) - control response

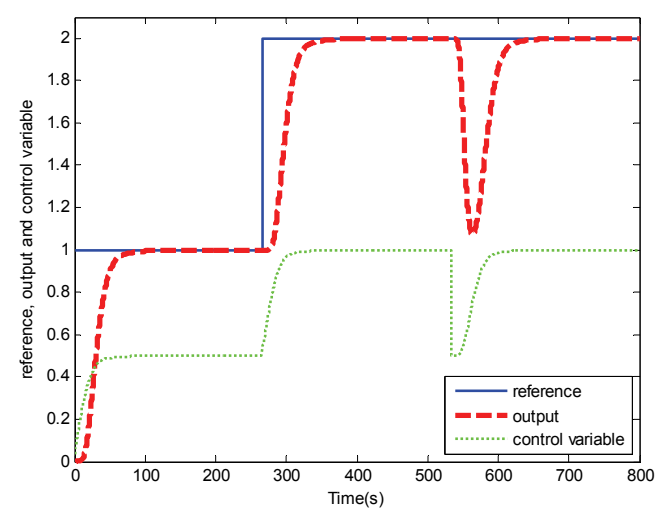

Figure 12: SOPDT model (34) - control response 
For both models, controller parameters were generated with the same parameter $m=0.15$. Naturally, for control simulations the original system (32) was utilized. The importance and relevance of the order approximation is obvious and apparent. A higher order in Figure 12 exhibit very smooth and acceptable control behavior.

\section{CONCLUSION}

An autotuning method using an asymmetric relay feedback test is studied. The identification procedure yields three process parameters for aperiodic first or second order transfer functions. After the identification relay experiment, controller parameters are designed using linear diophantine equation in the ring of proper and stable rational functions. This algebraic approach for a traditional 1-DOF feedback structure gives a class of PI or PID controllers. The pole placement principle in the methodology brings a scalar positive "tuning knob" for additional controller tuning. A Matlab, Simuling program implementation was developed for simulation and verification of the studied approach. Two illustrative examples support simplicity and efficiency of the proposed methodology.

\section{ACKNOWLEDGMENT}

This work was supported by the grant of Ministry of Education, Youth and Sports of the Czech Republic, MSM 7088352102.

\section{REFERENCES}

Åström, K.J. and T. Hägglund 1984. "Automatic tuning of simple regulators with specification on phase and amplitude margins," Automatica, vol. 20, pp. 645-651.

Åström, K.J. and T. Hägglund 1995. PID Controllers: Theory, Design and Tuning. Research Triangle Park, NC: Instrumental Society of America.

Gorez, R. and P. Klán 2000. "Nonmodel-based explicit design relations for PID controllers," in: Preprints of IFAC Workshop PID'00, pp. 141-146.

Hang, C.C., K.J. Aström and Q.G. Wang 2001. "Relay feedback auto-tuning of process controllers - a tutorial review" In Journal of Process Control 12, pp. 143-162.

Ingimundarson, A. and T. Hägglund 2000. "Robust automatic tuning of an industrial PI controller for dead-time systems" In: Preprints of IFAC Workshop PID'00, 149154.

Kaya, I. and D.P. Atherton 2001. "Parameter estimation from relay autotuning with asymmetric limit cycle data," in: Journal of Process Control, pp. 429-439.

Kučera, V. 1993. "Diophantine equations in control - A survey," Automatica, Vol. 29, pp. 1361-75.

Majhi, S. and D.P. Atherton 1998. "Autotuning and controller design for unstable time delay processes" In: Preprints of UKACC Int. Conf. on Control, 769-774.

Morilla, F., A. Gonzáles and N. Duro 2000. “Auto-tuning PID controllers in terms of relative damping" In: Preprints of IFAC Workshop PID'00, 161-166.

Pecharromán, R.R. and F.L. Pagola 2000. "Control design for PID controllers auto-tuning based on improved identification," in: Preprints of IFAC Workshop PID'00, pp. 89-94.
Prokop, R. and J.P. Corriou 1997. "Design and analysis of simple robust controllers," Int. J. Control, Vol. 66, pp. 905-921.

Prokop, R., P. Husták, and Z. Prokopová 2002. "Simple robust controllers: Design, tuning and analysis," in: Preprints of 15th IFAC World Congress.

Thyagarajan, T. and Ch.Ch. Yu 2002. "Improved autotuning using shape factor from relay feedback," in: Preprints of IFAC World Congres.

Vidyasagar, M. 1987. Control system synthesis: a factorization approach. MIT Press, Cambridge, M.A.

Vítečková, M. and A. Víteček 2004, "Experimentální identifikace metodou relé,“ in: Automatizácia a informatizácia.

Vyhlídal, T. 2000. “Anisochronic first order model and its application to internal model control," in: ASR '2000 Seminar.

Yu, Ch.Ch. 1999. Autotuning of PID Controllers. Springer, London.

\section{AUTHOR BIOGRAPHIES}

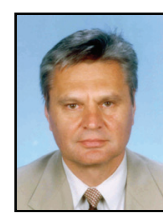

ROMAN PROKOP was born in Hodonin, Czech Republic in 1952. He graduated in Cybernetics from the Czech Technical University in Prague in 1976. He received post graduate diploma in 1983 from the Slovak Technical University. Since 1995 he has been at Tomas Bata University in Zlín, where he presently holds the position of full professor of the Faculty af Applied Informatics and a vice-dean of the faculty. His research activities include algebraic methods in control theory, robust and adaptive control, autotuning and optimization techniques. His email address is: prokop@fai.utb.cz.

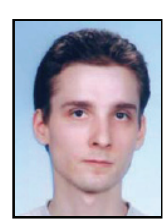

JIŘÍ KORBEL was born in Zlín, Czech Republic. He studied automatic control and informatics at the Tomas Bata University and graduated in 2004, now he is an post-graduate student and assistant at the Faculty of Applied Informatics in Zlín. His research activities include autotuning principles, algebraic and polynomial syntheses and modeling and simulations. His e-mail address is: korbel@fai.utb.cz.

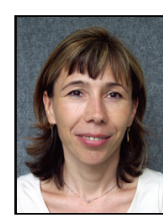

ZDENKA PROKOPOVÁ was born in Rimavská Sobota, Slovak Republic. She graduated from Slovak Technical University in 1988 (Automatic Control) and the $\mathrm{PhD}$ degree she received in Technical Cybernetics in 1993 from the same university. Since 1995 she been working in Tomas Bata University in Zlin, Faculty of Applied Informatics. She works there as an associate professor. Her main research activities include mathematical modeling, simulation, control of technological systems, programming and application of database systems. Her e-mail address is: prokopova@fai.utb.cz. 